\title{
Aesthetic Design Using Multi-Objective Evolutionary Algorithms
}

\author{
António Gaspar-Cunha ${ }^{1}$, Dirk Loyens ${ }^{2,3}$, and Ferrie van Hattum ${ }^{1}$ \\ 1 Institute of Polymer and Composites/I3N, University of Minho, Campus de \\ Azurém, 4800-058 Guimarães, Portugal \\ agc@dep.uminho.pt, fvh@dep.uminho.pt \\ http://www.dep.uminho.pt/agc/ \\ 2 School of Architecture, University of Minho, Campus de Azurém, 4800-058 \\ Guimarães, Portugal \\ 3 ESAD Escola Superior de Artes e Design, Avenida Calouste Gulbenkian, 4460-268 \\ Senhora da Hora, Matosinhos, Portugal \\ dirkloyens@gmail . com
}

\begin{abstract}
The use of computational methodologies for the optimization of aesthetic parameters is not frequent mainly due to the fact that these parameters are not quantifiable and are subjective. In this work an interactive methodology based on the use of multi-objective optimization algorithms is proposed. This strategy associates the results of different optimization runs considering the existent quantifiable objectives and different sets of boundary conditions concerning the decision variables, as defined by an expert decision maker. The associated results will serve as initial population of solutions for a final optimization run. The idea is that a more global picture of potential "good" solutions can be found. At the end this will facilitate the work of the expert decision maker since more solutions are available. The method was applied to a case study and the preliminary results obtained showed the potentially of the strategy adopted.
\end{abstract}

Keywords: aesthetic design, multi-objective evolutionary algorithms.

\section{Introduction}

Digital design culture and new paradigms in digital design thinking have a great impact on the design, development and realization of components and objects. Projects frequently embody a trade-off between multiple and interdependent requirements such as performance-related aspects, form freedom and complexity of the desired architectural expressions.

Current design methods, though already largely involving digital tools and processes, are not yet fully suited to dynamically optimize the design within its multiple boundary conditions. At the same time, conventional materials and technologies compromise the realization of the optimized design and its underlying concepts. Here, polymer and composite materials, in combination with their 
largely automated manufacturing methods, are a powerful group of materials to overcome this dilemma due to their inherent properties, such as aesthetically pleasing, lightness, ability to mould complex shapes, ease of fabrication and integration of parts.

In this research a computational method has been developed that can iteratively optimize a design towards its functional requirements using available design, simulation and user-interfacing tools. The method has been applied to the optimization of a 'generic' roof structure towards daylight conditions while minimizing area and thus weight and materials used. Multi-Objective Evolutionary Algorithms (MOEA) in combination with a decision making methodology have been used, together with critical Decision Maker (DM) interaction. The result indicates the usefulness of this model and the developed techniques in the early stage of the design process, leading to better design solutions.

This text is organized as follows: in section 2 the state-of-the-art concerning digital design methodology and the practical problem to be solved are presented; the problem characteristics as well the optimization methodology adopted are described in detail in section 3 ; in section 4 the methodology proposed is applied to an example and in section 5 the conclusions are stated.

\section{Digital Design Method}

\subsection{State of the Art}

Computers have been used in the design process for over fifty years. Initially the use of computers was limited to drawing, representation, basic structural analysis or construction planning. Eventually performance analysis was executed as an afterthought, but always as part of an essentially paper-based design process [1. It was not until the moment when design moved away from the conventional logic of representation and instead started interacting with the process of form generation itself, which we can speak of a new paradigm in the field of digital design [2. Since then digital design has evolved into a new and unique form of design.

The increasing integration of sophisticated and interactive digital design media throughout the complete design process, from early concept development until iterative testing and fabrication, has already provoked the emerging of new ways of design making and new ways of design thinking 34 . These concepts are starting to be the subject of research in the field of architecture. The concept of adaptation has been used to guide research towards the application of evolution-based generative design systems to shape architectural forms [5]. Other research has evolved in the development of specific software for methods combining structural grammars, performance metrics, structural analysis and stochastic optimization [6].

A compound model of digital design has been proposed as a future class of paradigmatic digital design [2]. These compound models are based on the integration of form finding, form generation, evaluation and performance processes. 
The development of processes or methods that aim to create new collaborative relationships between designer and computer, based on the idea of continuous feedback, are appointed as desired future research topics, though this research area is largely still in its infancy 617.

Although eminent architectural objects are today present, that have only been possible through the use of digital tools, the design processes used do not completely and interactively optimise at an early design stage [8]. At the same time, realisation has been frequently limited to the use of more traditional construction materials.

A desired design method would go beyond this 8 . The method should adopt processes that allow for dynamical multi-objective design optimization, integrated with - but not inherently limited to a sub-set of - available, both 'off-theshelf' and novel, material solutions. In construction process composite materials can play an important role, as they are known for their ability to combine the moulding of complex forms with varying, tailorable ranges of outstanding properties either aesthetic or structural, with a relative ease-of-processing and a large level of functional integration [9. Due to these characteristics, they have been a favorite material in design prototyping and final object manufacture, offering possibilities unable to be embodied in other materials. Furthermore, their fabrication can be highly automated, thus allowing for a high level integration with the desired digital design environment [10].

\subsection{Experimental}

The current research project explores new digital paradigms in a project development process within a framework of design as information processing rather than simple form finding. The project explores new relationships between the designer-as-toolmaker, information, process and the object. In this way the potential distinctive character of digital design thinking will be explored.

A new method is developed and tested, allowing integrating of complex quantitative and qualitative requirements at an early stage in the design process. This is achieved by combining multiple digital performance simulation tools with algorithms with generative capabilities, acting in the fuzzy front end of conceptual development. In this way, the design process is quicker and with more iterations, allowing complex functional and performance requirement integration and posing almost no limit to the freedom and complexity of forms and components used. As a first step, the method is applied to the fields of design, engineering and architecture, demonstrating that the existing design computing technologies, available and readily used in fields of architecture and composite technology, can open new territories for conceptual exploration.

For this purpose a generic roof structure geometry, represented by a single surface, was taken as a starting point (see Figure 1). This roof structure is represented by a single Non-Uniform Rational Basis Spline (NURBS) surface [11]. This method allows for a precise mathematical representation of a free form 
surface and for precise control by manipulating the control points of the surface. The control points determine the shape of the curve and a single control point only influences those intervals where it is active. This allows for the changing of one part of a surface while keeping other parts equal. The manipulation of control points is used in the everyday meaning of the word 'point', a location in $3 \mathrm{D}$ space defined by its coordinates.

In the present study a set of 20 control points were defined, allowing the virtually unlimited adaptation of the surface geometry. Based on the set of spatial coordinates of the control points, the surface is built in a general 3D design software [12. The area is calculated and the surface exported to a building analysis software 13 for subsequent numerical analysis, in this case the average daylight factor under the structure, as an indication of the light functionality of the structure. The results (Area, Daylight) are saved for subsequent use by the optimization routine, as described in the subsequent section.

The resulting optimized design combines both quantitative and qualitative evaluation of the design's performance, leading the exploration of a wider range of design solutions at an early stage in the concept phase. The best performing concept can then be used as the starting point for subsequent detailed design. The proposed model thus results in the streamlining of the design and development processes of architectural objects with a high degree of form freedom and system complexity. Applying this approach, architects and designers can conceive interactively, test the consequences of actions almost immediately, and explore different ways of solution refinements that are crucial in design and architecture.

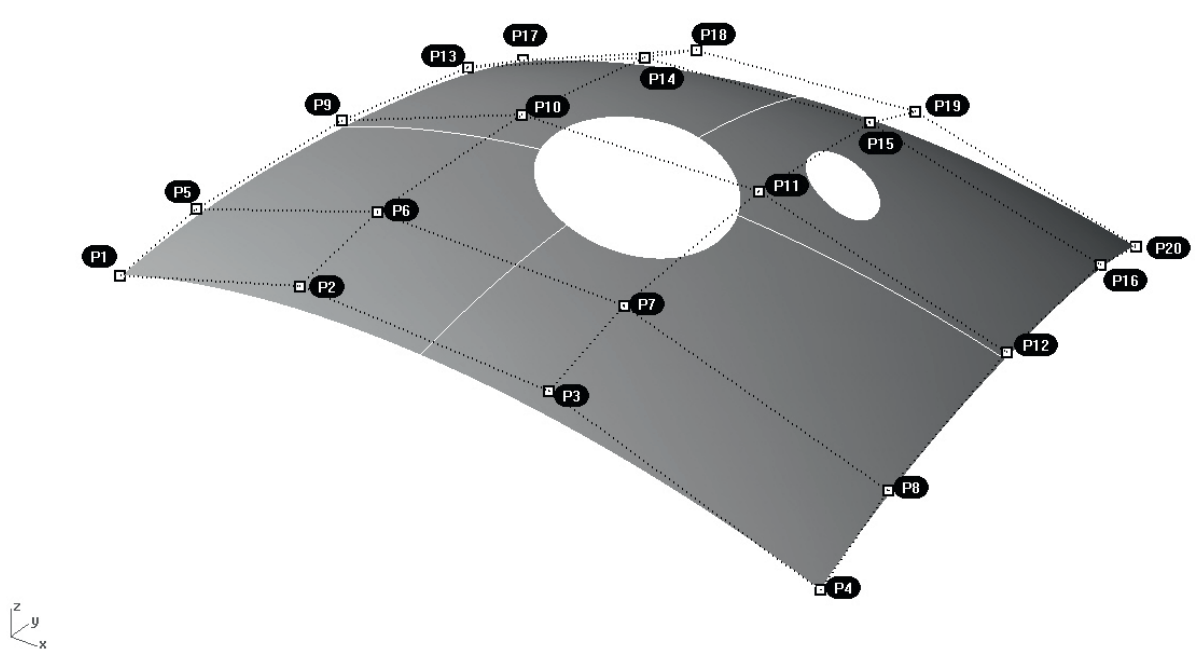

Fig. 1. Studied roof structure were the geometry is defined by the NURBS surface methodology (the limits for the coordinates of the control points are 5 meters) 


\section{Multi-objective Optimization}

\subsection{Problem Characteristics}

As mentioned above the problem to be solved has three objectives to be accomplished, the minimization of both Area and Daylight and the aesthetics design. The minimization of the structure Area, a measure of the effective use of material of 'lightness' of the structure, and the minimization of the Daylight under the structure, a measure of the effective 'functionality' of the structure, are two quantifiable objectives. Thus, they can be easily put up when using any MOEA to optimize the system. A trade-off between these objectives can be evidenced trough the generation of the Pareto front after optimization. The difficulty here concerns only with the interfaces between the software's used, i.e., the optimization routine (developed in house) and the 3D design and building analysis software (commercial softwares) used to calculate the objective values. Since these commercial software's do not run in background a specific interface approach based on Windows operating system scripts was implemented. This script simulates the use of the programs used.

Since the third objective is not quantifiable and, additionally is very subjective, a different strategy was adopted which takes into account the preferences of the DM involved. This can be seen as an iterative process: i) first the MOEA generates the Pareto fronts using the Area and Daylight objectives; ii) then, the DM selects the preferred regions taking into account aesthetics; iii) this information is inserted on the MOEA and new optimization is carried out. The process is repeated until a satisfactory solution is found by the DM.

Therefore, the resolution of this type of problems involves the articulation of preferences of a DM. In the present case the selection made by the DM, concerning one or more regions of the Pareto front, implies the definition of a measure of the relative importance of the objectives considered (in the present case two objectives exist, Area and Daylight). This can be better illustrated with the example of Figure 2. In region 1 the Area has more importance, since these solutions have better value for the Area, while in region 2 the Daylight is the most important objective (both objectives are to be minimized).

A traditional way to deal with multi-objectives consists in using an aggregation function, such as the weighted sum, were the relative importance of the various objectives are taking into account trough the definition of weights [14. In general terms three different classes of multi-objective preference methods can be identified, depending on how the search and decision processes are interconnected, a priori, a posteriori and iterative methods [15/16. In a priori methods, the DM must specify her or his preferences, expectations and/or options before the optimization process takes place. The preferences are expressed in terms of an aggregating function which combines individual criterion values into a single utility value. In the case of a posteriori method, after the generation of the Pareto optimal set, the DM selects the most preferred among the alternatives 


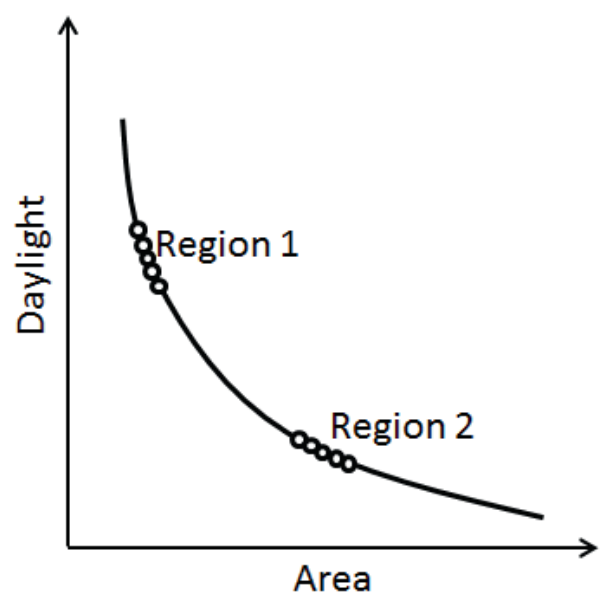

Fig. 2. Trade-off between Area and Daylight

taking into account his or her own preferences. Finally, in interactive methods the Decision making and the optimization processes occur at interleaved steps. At each step, partial preference information is supplied by the DM to the optimizer, which, in turn, generates better alternatives according to the information received.

Therefore, in the iterative methodology proposed different preferences methods are used (see Figure 3). At the beginning the MOEA runs without considering any preference and considering only the quantifiable objectives. After the Pareto front is generated, the DM selects the preferred region based on aesthetics parameters. The major difficulty consists in incorporating the information concerning the regions selected on the MOEA. The idea is to use a priori decision making methodology proposed before, which is based on the use of stress functions 17. In this method the incorporation of preferences is made through the definition of a set of weights quantifying the relative importance of the objectives. The value calculated for the stress function depend on the objective function itself as well of the weight chosen for this objective. The extension of the Pareto front found depend on the definition by the user of an algorithm parameter. For more details the reader is referred to 17. Starting from a population of solutions resulting from the previous optimization run the algorithm searches for solutions in the region corresponding to the weights chosen. However, care must be taken since the usability of interactive methods depends strongly on the extent to which the parameter values set by the DM as an expression of his or her preferences lead to solutions corresponding to those preferences.

Another important issue concerns the huge search space, which is a characteristic of this type of design problems (as will be seen on the problem tested below). In this case some of the solutions found, which are valid when calculating the Area and Light objectives, have some risk of not being valid concerning 


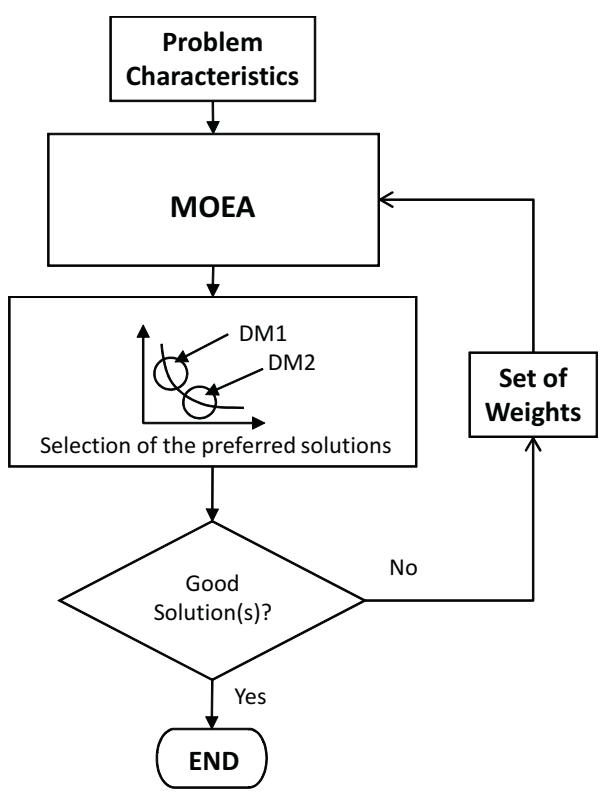

Fig. 3. Combination of a posteriori and iterative methods to select solutions in multiobjective problems involving aesthetic design variables

other questions such as possibility of fabrication. This aspect will not be deal in this phase of the work. However, a decision must be taken about the boundary conditions imposed to the decision variables. If the range of variation allowed is high the Pareto front obtained will have, certainly, solutions with very different aesthetics. If the range of variation is very restrictive, the possibility of losing some important designs (solutions) is high.

\section{Optimization Strategy}

In this section the strategy proposed to deal with the problems identified above, i.e., multiple objectives, non-quantifiable objectives and size of the search space, will be described in detail. The resolution of this type of problems can be made using three different situations:

Situation 1: The simplest situation consists in using the optimization algorithm (MOEA) without interacting with the DM (i.e., only one time). The DM defines the decision variables to be optimized and their range of variation and the objectives to be considered. Then, after running the MOEA, the DM selects the solutions from the pool of non-dominated solutions obtained using, for example, aesthetics criteria. In this case the DM must know very well the characteristics of the problem to be solved, since it is necessary to define beforehand the boundary conditions imposed to the design variables. 


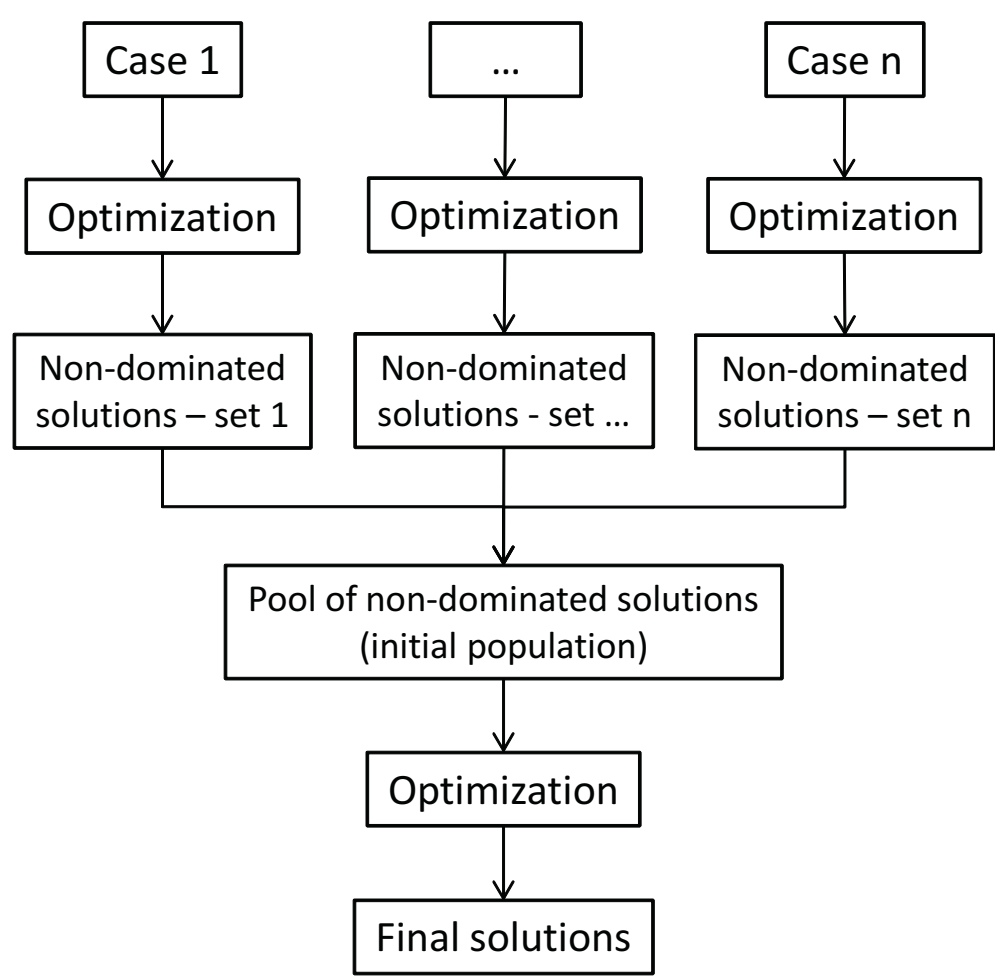

Fig. 4. Global structure of the optimization strategy adopted

Situation 2: This situation is illustrated in Figure 3. In this case an iterative process is pursued. In each interaction, new information (e.e., a set of weights) as provided by the DM, is taken into account. However, as in previous situation, the DM has to define the decision variables to be optimized and their range of variation and the objectives to be considered. Thus, the results produced will be certainly strongly dependent on the initial choice made by the DM.

Situation 3: This situation is illustrated in Figure 4. It starts by the definition of $n$ different cases, each one characterized by different set of restrictions (i.e., boundary conditions) imposed by the DM to the decision variables. Then, each one of these cases is optimized independently. At the end of this initial optimization step, the best solutions selected from all $n$ cases will be used to form a new population of solutions. This population will serve as initial population for the last optimization process. The optimization step in this case can be performed either using a simple MOEA optimization (as in situation 1) or using an iterative process (as in situation 2). It is expected that the non-dominated solutions found have characteristics taken from the different cases (i.e., different set of restrictions imposed). 


\section{Example of Application}

\subsection{Problem to Solve}

Different geometrical boundary conditions are input by the user, in order to explore different conceptual solutions. In the present work, 3 different geometrical boundary conditions (i.e., 3 different cases as represented in Figure 4) were used, each one leading to a different optimized subset of solutions. The surface was defined by 20 control points and defined by the NURBS method (see Figure 1). The natural light levels are calculated in Ecotect [13] over a horizontal analysis grid at ground level. The grid was formatted with a dimension of $5 \times 5$ meters and was set to a 5x6 matrix allowing for calculation over all 30 visible nodes. Calculations of natural light levels are neither time nor date dependant, so no parameters were specified and the default values of the software were used.

In case 1 , the less restrictive, the coordinates of the 20 control points represented in 1 (corresponding to 60 decision variables, the $3 \mathrm{D}$ coordinates of the control points) are allowed to vary between 0.5 and 5 meters. In case 2 the control points corresponding to the corners of the structure are fixed, i.e., points $\mathrm{P} 1(0,0,0), \mathrm{P} 4(5,0,0), \mathrm{P} 17(0,5,0)$ and $\mathrm{P} 20(5,5,0)$. In this case 48 decision variables are to be optimized. Finally, in the most restrictive case (case 3 ), the coordinates of the control points corresponding to the corners points as well to the border points are fixed, i.e., points $\mathrm{P} 1(0,0,0), \mathrm{P} 2(1.6,0,0.5)$, P3(0.338,0,0.5), P4(5,0,0), P8(5,0.65,0.18), P13(0,0.335,0.18), P16(5,0.335,0.18), $\mathrm{P} 17(0,5,0), \mathrm{P} 18(1.6,5.0,0.5), \mathrm{P} 19(0.338,5,0.5)$ and $\mathrm{P} 20(5,5,0)$. This corresponds to 24 decision variables. In cases 2 and 3 the coordinates of the remaining control points are allowed to range in the interval $[0.5,5]$ meters (as in case 1 ).

After this process the user is presented with the geometrical solutions and their performance, and allowed to bias the subsequent optimization step towards his/her preference (assumed to be based on the aesthetics of the solutions provided). The solutions selected are used as initial population for the final optimization. In this case no restriction to the decision variables are imposed, thus 60 decision variables are considered. They are allowed to range in the interval $[0$, $5]$ meters, the aim being to cover all possible solutions generated in the previous optimization cases.

The MOEA adopted in this work is the Reduced Pareto Set Genetic Algorithm (RPSGA) proposed before by one of the authors [1819. The values of the parameters inside the RPSGA are the best values as described in [19]. The main and elitist populations had 100 and 200 individuals, respectively; a roulette wheel selection strategy was adopted; a crossover probability of 0.8 , a mutation probability of 0.05 , a number of ranks of 30 and limits of indifference of the clustering technique of 0.01 were chosen. In all cases the algorithm ran only during 10 generations due to the computation time required by the modeling software.

\subsection{Optimization Results}

Figures 5 to 7 shows the initial population and the non-dominated solutions of the 10th generation, as well 3 different optimized designs of the roof structure, for 


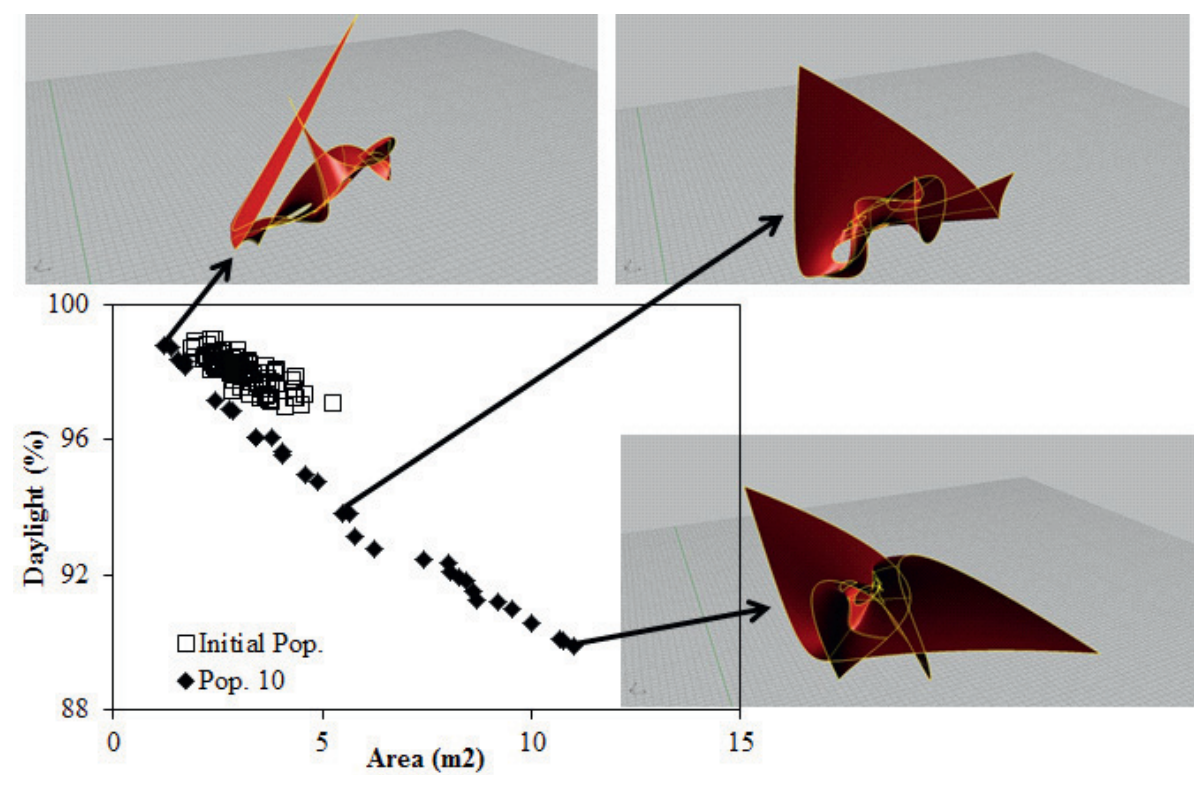

Fig. 5. Pareto frontier for case 1

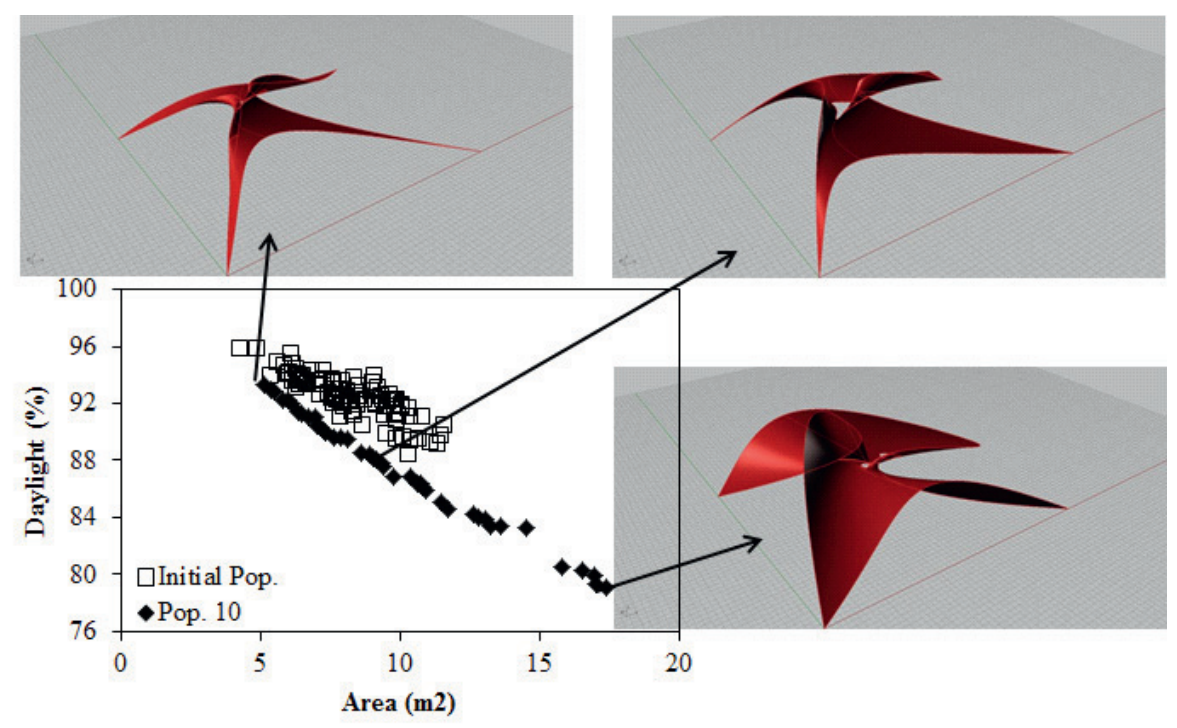

Fig. 6. Pareto frontier for case 2 


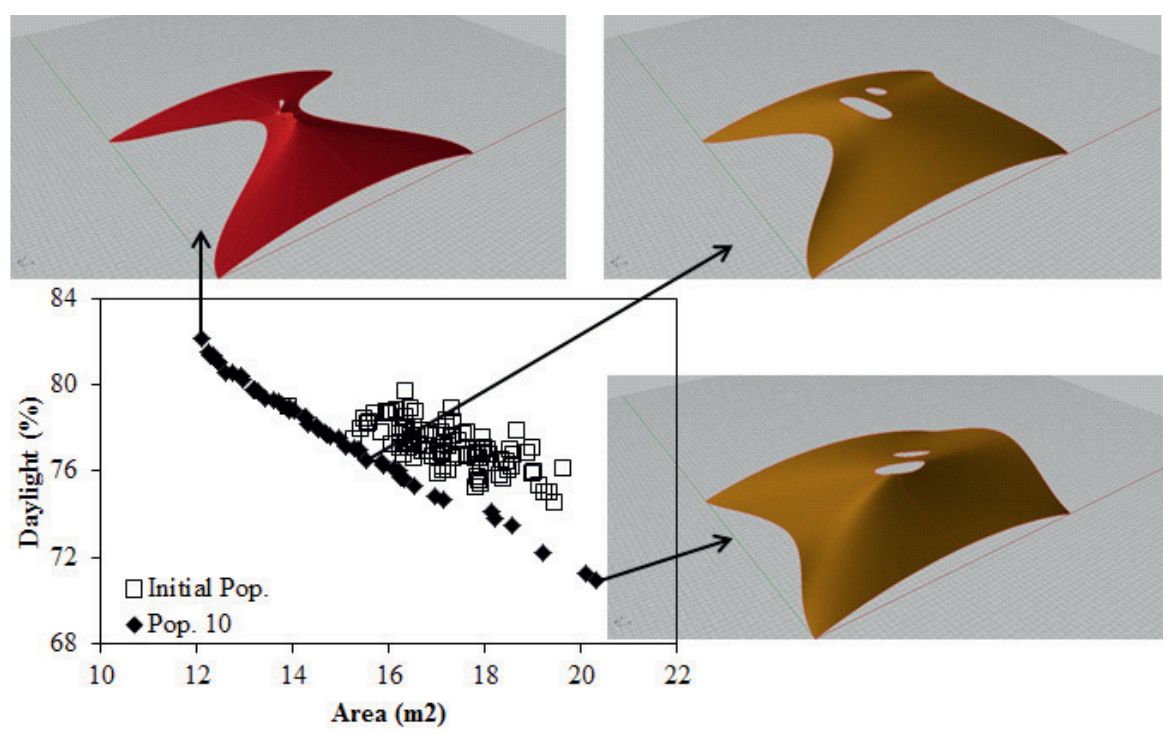

Fig. 7. Pareto frontier for case 3

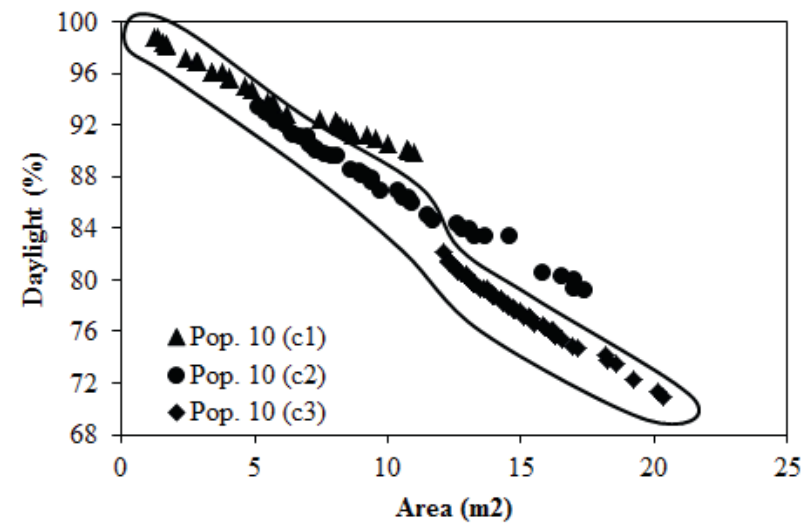

Fig. 8. Initial population for final optimization (non-dominated solutions of cases 1 to 3 )

cases 1 to 3, respectively. As can be seen the algorithm is able to evolve during the 10 generations and the Pareto frontier obtained in each case is well distributed. As expected, the roof structures obtained in case 1 are very random, while in the other two cases the structures obtained are coherent with the boundary conditions defined. In case 2 the corners are well defined and in case 3 this is also true for the four sides of the structure.

From the Pareto solutions of these three cases a new Pareto front was defined as illustrated in Figure 8. This set of solutions was the initial population of 


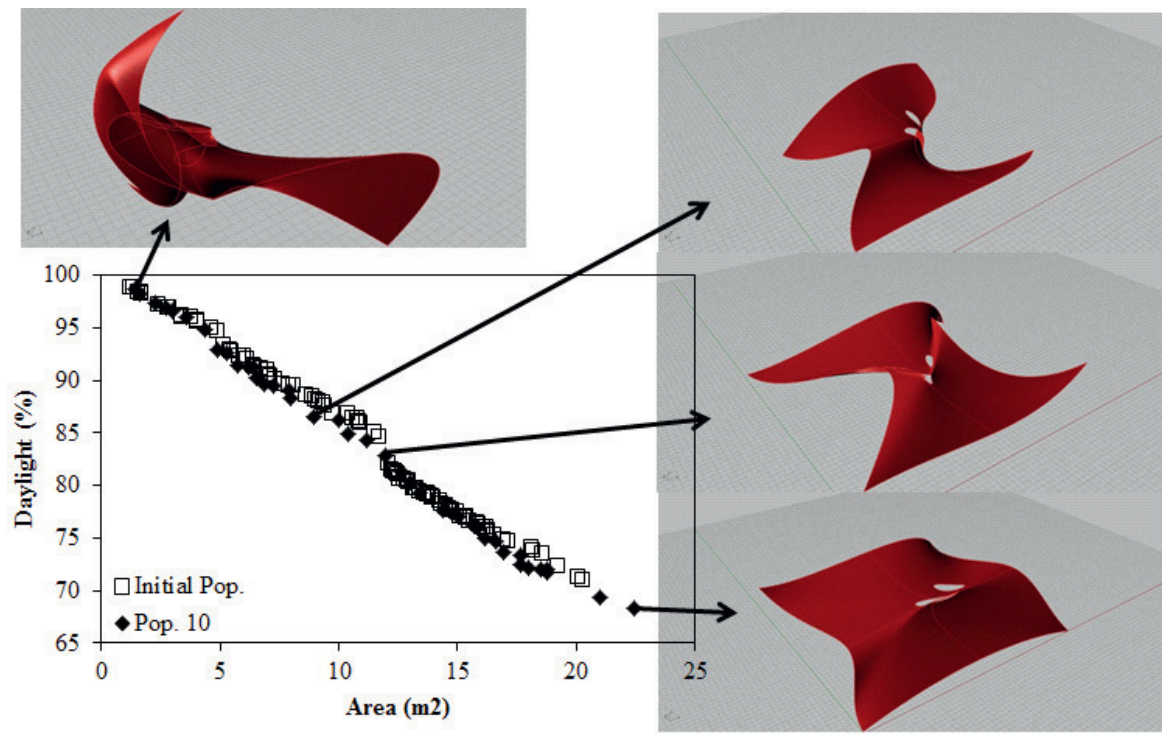

Fig. 9. Global optimization: initial population and non-dominated solutions after 10 generations

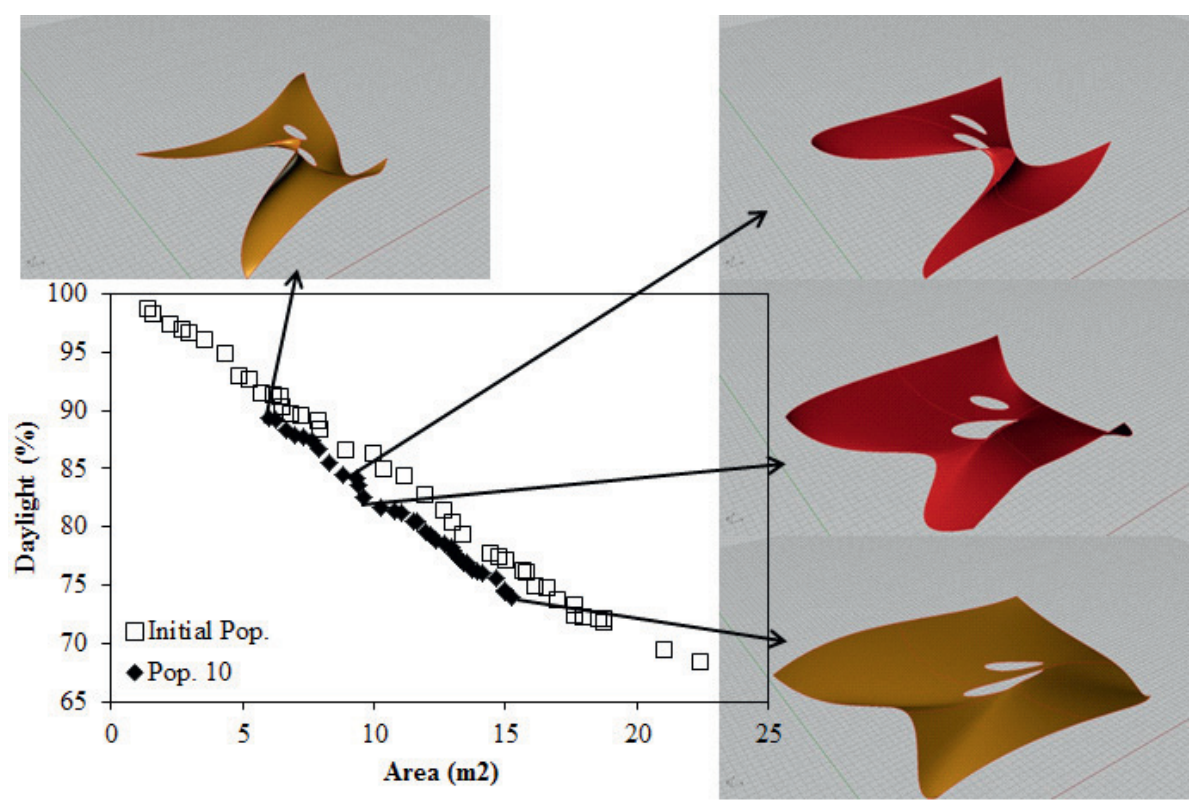

Fig. 10. Optimization considering a weight vector of $(0.5 ; 0.5)$ and as initial population the population resulting from previous run (Figure 9) 
the last optimization process, as identified in global strategy adopted in this work (Figure 44). It is interesting to note that the Pareto solutions of the three previous runs were able to define almost a continuous line. As can be observed in the optimization results presented in Figure 9 the MOEA was able to fill some of the gaps between the solutions of the initial population and some improvements are obtained. As expected, the new non-dominated solutions are able to cover all type of geometries obtained in the first three runs. Now the DM has the starting point for selecting the preferred geometries having a very good idea of their performance in terms of the two quantifiable objectives defined initially. This is done in Figure 10 where a set of weights of $(0.5,0.5)$ was selected by the DM, assuming that he/she "likes" the designs present in the center of the Pareto front (Figure 9). The decision making methodology based on the stress function is applied using as initial population the population found before. This method was able to obtain much better solutions than the previous ones and, simultaneously, converge for the preferred region of the Pareto front. At this point the DM can continues the process by selecting a new set of weights and/or by imposing additional restriction on the size of the portion of the Pareto front to be obtained.

Finally, it is important to note that in the generic roof structure two "skylights" were designed. Those "skylights", besides providing more light under the structure, are a fundamental design characteristic of this object, and contribute to the aesthetic perception of the geometry. But formal characteristics of a surface can change dramatically under the manipulation of the control points. As a result many of the intermediate solutions, although performing much better on the quantitative evaluation criteria, will not classify as aesthetically pleasing and will therefore be discarded or excluded by the DM from the next pool of solutions.

\section{Conclusions}

Design is about decision making and requires judgment and trade-offs based on the best available information. Therefore the role of optimization in design is to provide the designer with quantitative and qualitative information. This information is a way for increasing the designers understanding of the design problem and the nature of good solutions.

Design decisions made in the early stages of the design process have a higher effect on the final performance and outcome compared to decisions taken at later stages of the design process. Therefore the strategies which are followed in the beginning of a design project and the decisions made during those early stages are most important. Generative systems are an essential part of the future development of performative architectural systems where evolutionary principals are applied in the initial stages of the design process with the intent to automate explorative research. The outcome of those processes is expected to be surprising and inspiring. 
This study has introduced the use of a MOEA in the conceptual phase of the design process. The applied strategy for the use of a MOEA allowed for the DM to iteratively control the outcome and steer the process to a personal aesthetical solution. The DM can rely less on intuition to solve complicated and conflicting design requirements and concentrate efforts on innovative and aesthetical pleasing results.

The next step in this research is to demonstrate the applied design method and this specific MOEA for the design of an architectural object which can be tested and validated in the real physical world. In addition the method could be further developed and prepared for general use by less computer literate architects and designers for deployment in real world design processes.

Acknowledgements. One of the authors acknowledges the financial support received by the Portuguese Science Foundation under grant SFRH/BD/44600/ 2008.

\section{References}

1. Reffat, R.M.: Computing in architectural design: reflections and an approach to new generations of CAAD. The Journal of Information Technology in Construction 11, 655-668 (2006)

2. Oxman, R.: Theory and design in the first digital age. Design Studies 27, 229-265 (2006)

3. Kolarevic, B.: Architecture in the Digital Age: Design and Manufacturing. Spon Press, New York (2003)

4. Liu, Y.-T., Lim, C.-K.: New tectonics: a preliminary framework involving classic and digital thinking. Design Studies 27, 267-307 (2006)

5. Caldas, L.G.: An evolution-based generative design system : using adaptation to shape architectural form. PhD Thesis, Massachusetts Institute of Technology ( $\mathrm{Au}-$ gust 23, 2005)

6. Shea, K., Aish, R., Gourtovaia, M.: Towards integrated performance-driven generative design tools. Automation in Construction 14, 253-264 (2005)

7. Kilian, A.: Design exploration through bidirectional modelling of constraints. $\mathrm{PhD}$ Thesis, Massachusetts Institute of Technology (August 25, 2006)

8. Kolarevic, B., Malkawi, A.M.: Performative Architecture: Beyond Instrumentality. Spon Press, London (2005)

9. van Hinte, E., Beukers, A.: Lightness: The Inevitable Renaissance of Minimum Energy Structures, 3rd edn., Uitgeverij, Rotterdam (1998)

10. Sass, L., Oxman, R.: Materializing design: the implications of rapid prototyping in digital design. Design Studies 27, 325-355 (2006)

11. Rogers, D.F.: An Introduction to NURBS with Historical Perspective. Morgan Kaufmann, London (2001)

12. Robert McNeel \& Associates, www.rhino3d.com (accessed in 2010)

13. Autodesk - Ecotect, www.autodesk.com/ecotect (accessed in 2010)

14. Deb, K.: Multi-objective Optimization using Evolutionary Algorithms. Wiley, Chichester (2001) 
15. Miettinen, K.M.: Nonlinear Multiobjective Optimization. Kluwer, Boston (1999)

16. Hwang, C.L., Masud, A.S.: Multiple Objective Decision Making - Methods and Applications. Springer, Berlin (1979)

17. Ferreira, J.C., Fonseca, C.M., Gaspar-Cunha, A.: Assessing the quality of the relation between scalarizing function parameters and solutions in multiobjective optimization. In: IEEE Congress on Evolutionary Computation (IEEE CEC 2009), Trondheim, Norway (2009)

18. Gaspar-Cunha, A.: Modeling and Optimization of Single Screw Extrusion. PhD Thesis, University of Minho, Portugal (2000)

19. Gaspar-Cunha, A., Covas, J.A.: RPSGAe-A Multiobjective Genetic Algorithm with Elitism: Application to Polymer Extrusion. In: Metaheuristics for Multiobjective Optimisation. Lecture Notes in Economics and Mathematical Systems. Springer, Heidelberg (2004) 